\title{
Low Detection Rate of Multidrug-Resistant and Rifampicin-Resistant Tuberculosis in the Democratic Republic of Congo: Trend Analysis 2013-2017
}

\author{
Innocent Kashongwe Murhula ${ }^{1,2}$, Fina Mawete ${ }^{2}$, Lay Ofali ${ }^{2}$, Michel Kaswa ${ }^{2}$, \\ Francois Lepira Bompeka1, Jean Marie Kayembe Ntumba',2, Zacharie Kashongwe Munogolo1,2 \\ ${ }^{1}$ School of Medicine, Kinshasa University Hospital, Kinshasa, Democratic Republic of Congo \\ ${ }^{2}$ National Tuberculosis Program, Kinshasa, Democratic Republic of Congo \\ Email: innocent.kashongwe@unikin.ac.cd
}

How to cite this paper: Murhula, I.K., Mawete, F., Ofali, L., Kaswa, M., Bompeka, F.L., Ntumba, J.M.K. and Munogolo, Z.K. (2019) Low Detection Rate of Multidrug-Resistant and Rifampicin-Resistant Tuberculosis in the Democratic Republic of Congo: Trend Analysis 2013-2017. Journal of Tuberculosis Research, 7, 212-219.

https://doi.org/10.4236/jtr.2019.74020

Received: August 26, 2019

Accepted: November 5, 2019

Published: November 8, 2019

Copyright $\odot 2019$ by author(s) and Scientific Research Publishing Inc. This work is licensed under the Creative Commons Attribution International License (CC BY 4.0).

http://creativecommons.org/licenses/by/4.0/

\begin{abstract}
The Democratic Republic of Congo is one of the countries with a high prevalence of multidrug-resistant tuberculosis (MDR-TB) in Africa and worldwide. This study aims to describe the trends and level of screening since 2013. This is a retrospective descriptive study based on quarterly reports from the provinces. The number of cases, past histories, age, sex and HIV Co-infection was identified. It emerges that the number of cases increases significantly but does not reach the forecasts yet ( 912 cases of MDR/RR and XDR diagnosed in 2017 out of 3948 cases expected with a gap of 77\%). Patients with a history of TB predominate (63.5\% in 2017); but the proportion of patients with no history of $\mathrm{TB}$ increases $(36.5 \%$ in 2017). This suggests high rates among contacts. Women (37.8\%) and children under 15 (2.7\%) are less represented. TB/HIV Co-infection is $12 \%$ as for sensitive cases. Efforts still need to be made to improve screening.
\end{abstract}

\section{Keywords}

MDR-RR TB, Trends Screening, DRC

\section{Introduction}

Drug resistance to first- and second-line TB drugs represents a major challenge for overall control of TB [1] [2] [3] [4]. For 2015, the World Health Organization (WHO) estimated that 480,000 new cases of tuberculosis and approximately $14 \%$ of previously treated cases had drug-resistant TB strains; only 132,120 cases 
were reported [2]. The best estimate is that, worldwide in 2017, 558,000 patients (range 483,000 - 639,000) developed TB that was resistant to Rifampicin (RR-TB) and of these, $82 \%$ had multi-resistant TB form (MDR-TB) with resistance to at least Rifampicin and Isoniazid [5]. The exact estimate of the incidence of this infection remains difficult because of variations in strategies and reporting across different countries. But three countries accounted for almost half of the world's cases of MDR/RR-TB: India (24\%), China (13\%), Russian Federation (10\%) [3] [5]. Globally, 3.5\% of new TB cases and $18 \%$ of previously treated cases had MDR/RR-TB in 2017 [3]. Among cases of MDR/RR-TB, 8.5\% were estimated to have extensively drug-resistant TB (XDR), in which there is also associated fluoroquinolone and/or second-line injectables (kanamycin, amykacin, capreomycin) resistance [3].

The Democratic Republic of Congo (DRC) is one of 30 countries that bear the burden of TB in the world; it is among the 27 leading countries for the drug-resistant form and the top 4 in Africa with South Africa, Nigeria and Ethiopia [5]. The National resistance survey conducted in 2015 showed that $2.17 \%$ of new cases of pulmonary tuberculosis and $15.82 \%$ of retreatment cases were resistant to rifampicin and isoniazid [6]. Annual statements are far from WHO projections. This study seeks to describe trends in detection of RR/TB and MDR-TB in DRC between 2013 and 2017. At this period, the country began a new strategy with implementation of Gene Xpert equipment (Xpert MTB/RIF, Cepheid, USA) in all the 26 provinces. The main purpose is to contribute to the control of the disease in a context of limited resources.

\section{Material and Method}

This is a descriptive retrospective study. It is based on provincial quarterly reports validated during the annual reviews of the National Tuberculosis Program (NTP) for the period 2013-2017. The data concern children and adults. The concepts used meet WHO definitions [5]. The term "Multidrug Resistant" (MDR) applies to patients with at least rifampicin and isoniazid resistance without the combination of quinolones or injectables [5]. Cases with isolated resistance to rifampicin (RR) without isoniazid are also reported as such because they are more likely to be MDR. Extensively Resistant Tuberculosis cases (XDR) are not reported.

As a strategy, samples from the alleged MDR [1] [4] [7] [8] are tested on GeneXpert (Xpert MTB/RIF, Cepheid.USA). The country has 84 sites with GeneXpert. Specimens with resistance to rifampicin are subjected to the Line Probe Assay (LPA-MTBDRplus-sl or Hain test) to test other molecules: Isoniazid, Fluoro-quinolone, injectables and thus exclude XDR tuberculosis. All samples are also sent for performing Löwenstein-Jensen culture. There are 2 sites with LPA and an operational laboratory for culture for the whole country.

Variables studied are: age, sex, HIV state, previous TB treatment, origin (province). 
The quarterly reports were collected by the Central data manager of the NTP. At this level annual synthesis is performed.

The descriptive statistics consisted of calculating the mean and standard deviation for the quantitative data and proportions (percentages) for the categorical data.

\section{Results}

\section{1) Trend in the Screening of Multidrug-Resistant and Rifampi-} cin-Resistant TB cases in Democratic Republic of Congo 2013-2017

Table 1 presents results by year compared to all cases of tuberculosis detected.

Overall, the number of tuberculosis cases detected has increased by $38 \%$. For drug-resistant forms, this is also influenced by the increase in the number of GeneXpert equipment across the country from 2013. In the past, only two provinces had the equipment (Kinshasa, South Kivu). Actually all the 26 provinces are covered.

2) Provincial grouping based on the number of cases detected in 2017:

In 2017, four provinces report $72 \%$ of MDR/RR-TB cases in the country: Kinshasa (32\%), Haut Katanga (15\%), Kongo Central (13\%), Kasai Oriental (12\%). The other 22 provinces share $28 \%$. The 4 provinces are considered as "Hot Spots" for drug-resistant TB. Figure 1 shows the prevalence by province.

3) Patients with MDR/RR -TB by Gender in DRC: 2013-2017:

Table 2 gives the distribution of MDR/RR-TB patients by sex and year. Women are fewer. The sex ratio $(\mathrm{M}: \mathrm{F})$ is $1.6: 1$.

4) Patients with MDR/RR-TB by age:

Mean-age is 35.5 (11.3) years. Eighty-two patients are aged 0 to 14 years (2.7\%). The group aged 15 years or more is predominant.

5) Previous history of tuberculosis

Patients with a history of tuberculosis predominate. However, the number of those without a history of tuberculosis is increasing substantially to double 2013 in 2017. Table 3 summarizes the data.

6) Co-infection TB-VIH

Analysis is based on 2017 data only. From 893 cases screened, 518 (58\%) were tested for HIV, 61 (12\%) were HIV positive. HIV testing depended on the availability of the device and reagents in the health facilities.

Table 1. Cases of MDR-TB and RR-TB in DRC: 2013-2017.

\begin{tabular}{cccc}
\hline Year & Total TB Cases $(\mathrm{n})$ & TB-MDR $(\mathrm{n})$ & TB RR (n) \\
\hline 2013 & 110,563 & 334 & 41 \\
2014 & 117,681 & 424 & 32 \\
2015 & 121,024 & 466 & 40 \\
2016 & 133,262 & 646 & 63 \\
2017 & 152,744 & 803 & 90 \\
TOTAL & 635,274 & 2673 & 266 \\
\hline
\end{tabular}


Table 2. MDR /RR-TB in DRCongo by sexe: 2013-2017.

\begin{tabular}{cccccc}
\hline Year & Total Cases $(\mathrm{n})$ & Male $(\mathrm{n})$ & $\%$ & Female $(\mathrm{n})$ & $\%$ \\
\hline 2013 & 375 & 213 & 56.8 & 162 & 43.2 \\
2014 & 456 & 284 & 62.3 & 172 & 37.7 \\
2015 & 506 & 341 & 67.4 & 165 & 32.6 \\
2016 & 709 & 434 & 61.2 & 275 & 38.8 \\
2017 & 893 & 555 & 62.1 & 338 & 36.9 \\
Total & 2939 & 1827 & 62.2 & 1112 & 37.8 \\
\hline
\end{tabular}

Table 3. History of TB in MDR and RR Patients 2013-2017.

\begin{tabular}{cccccc}
\hline \multirow{2}{*}{ Year } & Total Cases (n) & \multicolumn{2}{c}{ No TB History } & \multicolumn{2}{c}{ TB History } \\
\cline { 3 - 6 } & & $\mathrm{n}$ & $\%$ & $\mathrm{~N}$ & $\%$ \\
\hline 2013 & 375 & 55 & 14.7 & 320 & 85.3 \\
2014 & 456 & 90 & 19.7 & 366 & 80.3 \\
2015 & 506 & 110 & 21.7 & 396 & 78.3 \\
2016 & 709 & 126 & 17.8 & 583 & 82.2 \\
2017 & 893 & 326 & 36.5 & 567 & 63.5 \\
TOTAL & 2939 & 707 & 24.0 & 2232 & 75.9 \\
\hline
\end{tabular}

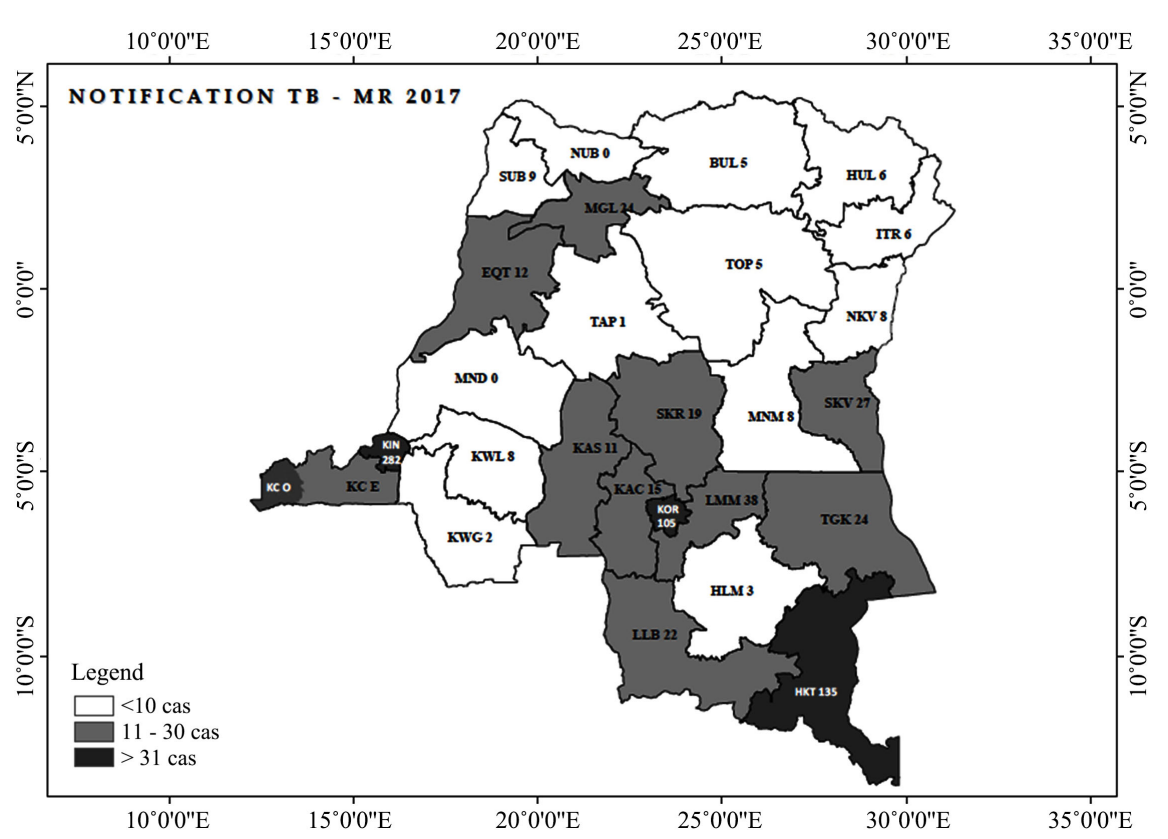

Figure 1. Prevalence of TB-MDR/RR in DR Congo by province. SUB = Sud Ubangi, NUB $=$ Nord Ubangi, BUL $=$ Bas Uélé, $\mathrm{HUL}=$ Haut Uélé, $\mathrm{ITR}=$ Iturie, $\mathrm{EQT}=$ Equateur, $\mathrm{MGL}$ $=$ Mongala, $\mathrm{TOP}=$ Tshopo, NKV $=$ Nord Kivu, TAP $=$ Tshwapa, $\mathrm{MND}=$ Mai-Ndombe, KAS $=$ Kasai, KAC $=$ Kasai Central, SKR $=$ Sankuru, KOR $=$ Kasai Oriental, HLM $=$ Haut Lomami, MNM = Maniema, SKV = Sud-Kivu, TGK = Tanganika, HKT = Haut Katanga, $\mathrm{LLB}=$ Lualaba, KWL $=$ Kwuilu, $\mathrm{KWG}=$ Kwango, $\mathrm{KCE}=$ Kongo Central Est, $\mathrm{KCO}=$ Kongo Central Ouest, KIN = Kinshasa, LMM = Lomami. The 4 Hot-Spot provinces are highlighted in black: Kinshasa, Kongo Central, Kasai Central and Haut Katanga. 


\section{Discussion}

DR Congo is among the 30 countries worldwide bearing the burden of the TB as endemic [2] [4] [5]. Drug-resistant forms are increasingly being detected. We have observed an increase in cases for both susceptible and drug-resistant TB between 2013 and 2017. This trend is expected to continue if we consider the expected prevalence of 250 cases of Tuberculosis/all forms per 100,000 inhabitants [4]. The current progress is based on initiatives such as The Global Fund for Tuberculosis, Malaria and HIV, Damien Action, Challenge TB etc.

However, the number achieved remains below the WHO estimates [7]. In 2017, there were 893 cases of MDR-RR TB and 19 XDR; that was below the expected number of 3948 cases [5]. The national survey on TB drug resistance conducted in 2015 found a prevalence of $2.17 \%$ among new cases and $15.82 \%$ for retreatment cases [6]. This rate remains lower than those described in other countries such as Angola and Ethiopia, where $8 \%$ and $4.1 \%$ are respectively reported in the group of new cases, and $71.1 \%$ and $19 \%$ respectively cases already treated for tuberculosis [9] [10] [11] [12] [13]. Urgent action is required to improve the coverage and the quality of diagnosis and treatment for people suspected to be infected by resistant TB strains. Closing gaps in detection and treatment requires much higher coverage of drug susceptibility testing among TB patients, reducing underdiagnosis of TB, improving access on new diagnosis tools and better organization of health system [8] [14].

MDR/RR TB patients with a history of tuberculosis account for two-thirds. This is usual. Results reported in other countries are similar [9] [12] [15] [16]. It should be noted, however, that there is an increase in the number of patients without a history of tuberculosis that can reflect the importance of contacts. Strengthening the strategy for mapping contacts is advised.

Four provinces screen $72 \%$ of MDR-RR TB cases in the country. They are considered as Hot Spots for drug-resistant TB. Two have areas with intense mining activities. For the 2 others, it is a big city and a harbor with intense activity. This context requires innovative approaches to manage particular patients such as mine workers, harbor residents and crowded cities with often comorbidities [17].

Women are fewer. This is seen even for sensitive TB. Marçôa D. et al. report a sex ratio of 1.6:1 for men [18]. On the one hand, they mention the difficulties women in resource-poor countries face in accessing health facilities. On the other hand, men have comorbidities that may expose them to tuberculosis and phamaco-resistant forms; include alcohol, tobacco, imprisonment, and HIV. In women, the overall prevalence of tuberculosis reported remains lower than in men and this has been reported for many infectious diseases. Interactions between sex hormones and the immune system are thought to be implied in the differences observed.

Patients younger than 15 years represent only $2.7 \%$. Overall, children are less numerous for both sensitive TB and drug-resistant forms [4] [5]. Several factors 
challenge confirmation of TB among children: low bacillary load, difficulty coughing, and high frequency of extra-pulmonary forms requiring more specialized investigations [17]. Algorithms are available but they require capacity building for the staff providing care.

Co-infection with HIV is found in $12 \%$ of patients in this study. This percentage is similar to that found in sensitive TB cases [19]. Although HIV infection is recognized as a potent risk factor for developing $\mathrm{TB}$ in a person with latent $\mathrm{TB}$, it is not formally recognized as risk factor for the development of drug-resistant TB. [17] Person living with HIV will develop both sensitive and resistant TB. However, during treatment with ARVs, some adverse effects may alter the absorption of antituberculosis drugs or lead to dysfunction that may lead to drug resistance.

\section{Conclusion}

The detection of MDR/RR TB cases in DRC has been increasing since 2013. However, the gap to be covered compared to projections remains high. This can be explained by the difficult access to diagnostic sites. The small number of women and children requires an appropriate approach in the epidemiological context. Strengthening teams to identify suspects, supporting the dispatch network of samples from health centers to diagnostic laboratories and increasing the number of laboratories for performing culture and LPA will significantly improve screening.

\section{Limitations of This Study}

As retrospective study, it was not easy to have information about contacts tracking and so answer the progressing rate of new cases among MDR/RR TB diagnosed. Also, the feedback from the main laboratories to the health structures was not always given to certify the results. These aspects will be corrected by a cohort study now undergoing.

\section{Authors Contributions}

Innocent Kashongwe Murhula: Conception, data organization and manuscript management;

Fina Mawete: collection of data from national MDR/ RR and XDR TB reports, manuscript management;

Lay Ofali: data collection from TB susceptible report;

Michel Kaswa: Supervision of data collection;

Francois Lepira Bompeka: reviewer of manuscript;

Jean Marie Kayembe Ntumba: Manuscript Management;

Zacharie Kashongwe Munogolo: Coordination of study.

\section{Conflicts of Interest}

The authors declare no conflicts of interest regarding the publication of this paper. 


\section{References}

[1] Rudrajit, P. (2018) The Threat of Multidrug-Resistant Tuberculosis. Journal of Global Infectious Diseases, 10, 119-120. https://doi.org/10.4103/jgid.jgid_125_17

[2] World Health Organization (2016) WHO Treatment Guidelines for Drug Resistant Tuberculosis: 2016 Update. World Health Organization, Geneva.

[3] Falzon, D., Mirzayev, F., Wares, F., Baena, I. G., Zignol, M., Linh, N., Raviglione, M., et al. (2015) Multidrug-Resistant Tuberculosis around the World: What Progress Has Been Made? The European Respiratory Journal, 45, 150-160. https://doi.org/10.1183/09031936.00101814

[4] World Health Organization (2013) Global Tuberculosis Report 2013. WHO, Geneva.

[5] World Health Organization (2018) Who Treatment Guidelines for Multidrug and Rifampicin-Resistant Tuberculosis, 2018 Update. World Health Organization, Geneva.

[6] National Tuberculosis Program, DR Congo (2018) National Drug Resistance Survey Report.

[7] World Health Organization (2019) WHO Consolidate Guidelines on Drug-Resistant Tuberculosis Treatment. World Health Organization, Geneva. https://creativecommons.org/licenses/by-nc-sa/3.0/igo

[8] Pontali, E., D’Ambrosio, L., Centis, R., Sotgiu, G. and Migliori, G.B. (2017) Multidrug-Resistant Tuberculosis and beyond: An Updated Analysis of the Current Evidence on Bedaquiline. European Respiratory Journal, 49, Article ID: 1700146. https://doi.org/10.1183/13993003.00146-2017

[9] Caminero, J.A. (2013) Guidelines for Clinical and Operational Management of Drug-Resistant Tuberculosis. International Union against Tuberculosis and Lung Disease, Paris, France, 47-53

[10] Aznar, M.L., Rando-Segura, A., Moreno, M.M., et al. (2019) Prevalence and Risk Factors of Multidrug-Resistant Tuberculosis in Cubal, Angola: A Prospective Cohort Study. The International Journal of Tuberculosis and Lung Disease, 23, 67-72. https://doi.org/10.5588/ijtld.18.0231

[11] Asgedom, S.W., Teweldemedhin, M. and Gebreyesus, H. (2018) Prevalence of Multidrug-Resistant Tuberculosis and Associated Factors in Ethiopia: A Systemic Review. Journal of Pathogens, 2018, Article ID 7104921. https://doi.org/10.1155/2018/7104921

[12] Tessema, B., Beer, J. Emmrich, F., Sack, U. and Rodloff, A.C. (2012) First- and Second Line Antituberculosis Drug-Resistance in Northwest Ethiopia. The International Journal of Tuberculosis and Lung Disease, 16, 805-8011. https://doi.org/10.5588/ijtld.11.0522

[13] Seung, K.J., Keshaviee, S. and Rich, M.L. (2015) Multidrug-Resistant Tuberculosis and Extensively Drug-Resistance Tuberculosis. Cold Spring Harbor Perspectives in Medicine, 5, a017863. https://doi.org/10.1101/cshperspect.a017863

[14] Caminero, J.A. (2013) Guidelines for Clinical and Operational Management of Drug-Resistant Tuberculosis. International Union Against Tuberculosis and Lung Disease, Paris, France, 7-12

[15] Kibret, K.T., Mages, Y., Meniah, P. and Biadgiligu, S. (2017) Treatment Outcomes for Multidrug-Resistant Tuberculosis under DOTS-Plus: A Systemic Review and Meta-Analysis of Published Studies. Infectious Diseases of Poverty, 6, 7. https://doi.org/10.1186/s40249-016-0214-x 
[16] Iseman, M.D. (1993) Treatment of Multidrug-Resistant Tuberculosis. The New England Journal of Medicine, 329, 784-791. https://doi.org/10.1056/NEJM199309093291108

[17] Caminero, J.A. (2013) Guidelines for Clinical and Operational Management of Drug-Resistant Tuberculosis. International Union against Tuberculosis and Lung Disease, Paris, France, 147-160.

[18] Marçôa, R., Ribeiro, A.I., Zao, I. and Duarte, R. (2018) Tuberculosis and Gender-Factors Influencing the Risk of Tuberculosis among Men and Women by Age Group. Pulmonology, 24, 199-202. https://doi.org/10.1016/j.pulmoe.2018.03.004

[19] Antunes, M.L., Aleixo-Dias, J., Atunes, A.F., Pereira, M.F., Raymundo, E., Rodrigues, M.F. (2000) Anti-Tuberculosis Drug Resistance in Portugal. The International Journal of Tuberculosis and Lung Disease, 4, 223-231. 